\title{
Joint Trajectory and Fatigue Analysis in Wheelchair Users
}

\author{
Maddalena Sebastiani ${ }^{1}$, Nicola Garau ${ }^{1}$, Francesco De Natale ${ }^{1}$, and Nicola Conci ${ }^{1}$ \\ ${ }^{1}$ University of Trento, Via Sommarive, 9, 38123 Povo,Trento TN \\ maddalena.sebastiani@studenti.unitn.it, \{nicola.garau,francesco.denatale,nicola.conci\}@unitn.it
}

\begin{abstract}
A successful rehabilitation process always requires both medical and infrastructural support. In this paper we focus on paraplegic wheelchair users, aiming at understanding the correlation between accuracy in guidance and muscular fatigue, while moving on a known training path. In particular, we study the trajectories performed and the corresponding shoulder forces distribution, while changing the inclination of the seat. At the motor level, the point of interest is the shoulder, as, in the prolonged use of wheelchairs with manual self-propulsion, it is generally source of pain. The objective is to demonstrate that there is a potential relationship between trajectory discontinuities and shoulder pain, and foster the development of best practices aimed at preventing the raise of shoulder-related pathologies, by correcting the users movements and the wheelchair setup. This project is meant to be a first study of the subject, so far little addressed, and is not meant to be a clinical study. The experiments have been conducted within the premises of the Living Lab AUSILIA and validated with the help of experienced medical personnel.
\end{abstract}

\section{Introduction}

People with motor disabilities, whether it is a permanent or temporary disability, often require continuous assistance. The project AUSILIA (Assisted Unit for Simulating Independent LIving Activities, Fig. $1 / 1$ is a living lab, aimed at improving the user capabilities, thanks to an advanced technological infrastructure able to measure the user's skills and needs, recommending the most suitable instruments to fostering autonomy and independent living. As described in [12], within the premises of AUSILIA, users perform small daily activities in a controlled environment simulating a real fully furnished house. In this way it is possible to observe what difficulties they would be facing once back home. The

\footnotetext{
${ }^{1}$ http://www.ausilia.tn.it
}

AUSILIA living lab consists of two main environments: the home apartment and the gym as shown in Fig. 1. Both the apartment and the gym are equipped with a multi-sensory infrastructure, necessary to capture the user motion and his/her interactions with the environment. The gym is the reference environment for this work. It is equipped with numerous platforms simulating different pavements and surfaces, in which wheelchair users can be monitored and their capabilities assessed. The goal of this paper is to jointly analyze trajectory and shoulder fatigue targeting paraplegic users, using manual self-propulsion wheelchairs, with different inclinations of the seat and in presence of obstacles in the route. The system is composed of two main modules: on the one hand we rely on a camera network for tracking and trajectory analysis; on the other hand we focus on the muscular activity through the positioning of an electromyograph and an electrogoniometer on the shoulder and arm of the user. The output of the analysis, conducted in synergy with the medical personnel, aims at highlighting a potential correlation between salient points in the trajectories and discontinuities in the shoulder movement. The data, as will be shown in the experiments section, is made available through an ad-hoc user interface, where doctors and caregivers can assess the motion and guiding abilities with reference to the specific wheelchair setup.

At the current stage of development, the presence of the electromyograph and an electrogoniometer is necessary mostly to acquire the ground truth, while in the future it is expected that the analysis is conducted by solely using video cameras and/or, more in general, non wearable sensors, for an improved user experience and limited invasiveness.

\section{Related work}

In this paper we focus on manual wheelchairs users, as many studies show the high correlation between the setup of the wheelchair and shoulder pain [10, 1, 6, 9, 7]. The literature shows how the force applied to push the wheelchair 


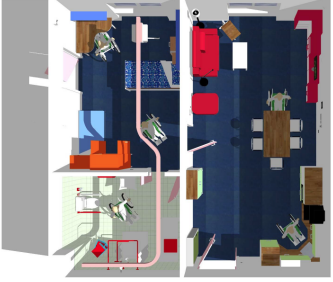

(a) Apartment

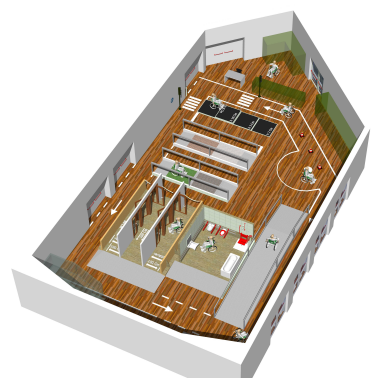

(b) Gym
Figure 1: Layout of the apartment and gym of the AUSILIA project.

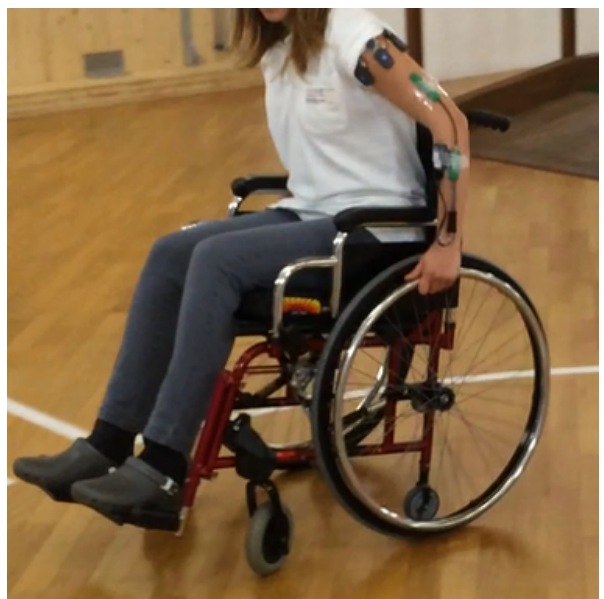

Figure 2: Image of a user testing all the described equipment: EMG sensors, electrogoniometer, and wheelchair.

relates to the specific type of movement performed and also to the presence of obstacles in the user's path, elements that can be well modeled and described through the trajectory performed. Studies carried out in this area cover different topics. The authors in [6] clearly highlight how the raise of shoulder pain in wheelchairs users during daily activities is a widespread phenomenon, so that the authors, as a final goal outcome of their study, define the Wheelchair User's Shoulder Pain Index (WUSPI). Using this index, they highlight an increase in pain in quadriplegic users than paraplegic users above all, in relation to increasing age [1].

\subsection{Human detection and trajectory analysis}

As far as the trajectory analysis is concerned the literature is very rich. Most of the approaches focus on purely vision-based approaches, which keep the implementation and deployment costs low, thus not requiring the adoption of expensive wearable sensors. The most straightforward solutions are based on background subtraction techniques for blob detection, followed by tracking [15, 13]. How- ever, background subtraction is generally not robust, and tracking-by-detection algorithms are generally preferable [3]. From the tracking perspective, the analysis is generally conducted on the 2D trajectory. Many different solutions have been proposed. We will adopt the methods proposed by Piotto et al. [11], in which the authors explore the analysis and comparison of trajectory subsets: after subdividing the trajectory into short segments, they look for temporal discontinuities, as well as spatial discontinuities in terms of sharp direction variations.

A recent trend in computer vision is to estimate the human pose through deep networks. Among the most cited papers in the recent literature, we can find [5], an approach for real-time 2D multi-person pose estimation, which serves as a basis for the popular framework OpenPose [4]. 2D human pose estimation has become popular thanks to its robustness towards human (self-)occlusions. Moreover, pose estimation has established a new baseline in terms of performances and detection accuracy especially for what concerns body joint detection, making it a perfect candidate for realtime trajectory analysis applications. Moreover, a skeleton (resulting by the connection of the extracted joints) can provide a much better semantic representation of a person, especially if compared to a bounding box resulting from a $2 \mathrm{D}$ blob. As an example, Zimmermann et al. [17] trace the user's movements through 2D skeleton extraction to teach robots, how to help the user perform the actions he is willing to perform.

\subsection{EMG signals analysis}

The electromyographic (EMG) signal is used to characterize the activity of a muscle. The activation of a particular muscle can be both voluntary and involuntary [2, 8, 14] These signals are very complex to handle, since they resemble the combination of numerous stimuli and are generally affected by a considerably large amount of noise, thus requiring the adoption of signal processing techniques to highlight the meaningful signal components.

Although the applicable filters may vary depending on the analysis, an overall good method for cleaning a signal is proposed by [2]. This method considers four main steps: filtering, rectification, smoothing and features extraction. The filtering and smoothing steps are signal-dependent, because every signal is affected by different noise sources and can have different ranges, depending on the performed movement, as well as the type of muscle being activated. A more detailed explanation is available in [16], in which the authors have listed possible filtering strategies together with the corresponding references. An additional feature of the EMG signal analysis is that it can be performed both in the time and in the frequency domain, as underlined by [14, 2, 16] depending on the different physical parameters [16] analyzed. There are four fundamental physical param- 
eters that can be extracted from the temporal and frequency analysis, namely joint motion, force, velocity, and muscle fatigue. Those of greater interest, especially with regard to this work, are muscular force and fatigue. The first one, defined as force or muscle tension, can be extracted in the time domain analysis, due to the fact that it can be related to the amplitude of the EMG signal. In addition, for experiments with constant tension exercise, the average value of the signal that has been previously rectified, is a measure of tension. A feature widely used in literature to extract this value is the Root Mean Square. Instead, the muscle fatigue is generally measured analyzing the frequency domain, as it has been found that the fatigue produces changes in the EMG spectrum. In particular, an increase in fatigue decreases the high-frequency components of the spectrum.

\section{Proposed model}

Our focus is to jointly analyze wheelchair users' trajectories and the development of fatigue, mainly at shoulder level, in relation to their posture. The analysis has been conducted in the AUSILIA gym, by detecting sudden speed and direction changes in the path that can be linked to incorrect movements, to the presence of obstacles, such as steps, or to variations in the road inclination. For the user detection phase, we rely on the method described in [5] and [4], combined with a background subtraction motion model, that can support the algorithm is those cases, in which the skeleton detection fails, due to severe occlusions of the body details. For the shoulder and posture analysis instead, we adopt a mixed approach, that links data from the EMG sensor, the user's body pressure distribution, and pose data in terms of joints position. The main steps of the developed method are shown in Fig. 3

\subsection{Sensors positioning, calibration, and recording}

Four cameras have been installed on the gym perimeter so as to guarantee the coverage of the whole area of interest. The approximate coverage on the ground by each camera is shown in Fig. 4

Cameras are subject to distortions of various kinds and therefore require calibration, to determine both the intrinsic and extrinsic parameters. As we are working with a small camera network, it is also necessary to relate each camera to the others in the network, via the computation of the fundamental matrix, as well as linking the cameras with the real world through the computation of the homography matrix.

With the camera network fully calibrated, it is possible to start collecting data. Since it is not convenient, in terms of required storage space, to record all the videos from all the cameras in the gym simultaneously, the goal is to trigger the recording of each camera based on the user's position, automatically handling the hand-off. The final video consists then of the concatenation of the single views. How- ever, in order to guarantee that no relevant data is lost, the entire videos for each camera are also temporarily stored, until the medical personnel confirms that no salient event has been missed.

\subsection{Trajectory analysis through skeleton detection}

In order to determine the users' trajectory, the first step consists of determining the position of the skeleton. For this phase, we rely on the OpenPose library [4], which allows for a good detection also in presence of partial occlusions. Only in case the skeleton is not found, we backtrack to background subtraction, so as to ensure a continuous tracking over time (Fig. 6).

We can now define a bounding box that resembles the position of the users at a given time instant. At each frame $p_{G}$ represents the ground point. When the pose estimation succeeds, $p_{G}$ can be identified as the median point of the 11 th and 14th skeleton joints (heels joints). When pose estimation fails, $p_{G}$ is located at the lower median point of the bounding box obtained via background subtraction.

At this stage, the trajectory is still noisy and uneven. As a consequence, a filtering and smoothing phases are necessary to obtain a more uniform and meaningful trajectory. Therefore, we employ a two-step filtering approach: first, we apply a Kalman filter; we then refine the obtained trajectory with a moving average filter. The outcome of the trajectory filtering is shown in Fig. 7 .

All the extracted points that contribute to the definition of the trajectory can now be mapped to the ground plane in the real world coordinates thanks to the calibration procedure described above.

Velocity and acceleration are then extracted by computing the values over a one-second window, so as to cope also with minor irregularities that might occur at smaller time scales. In addition, the speed value for each frame is also used to graphically indicate on the map the exact points where a change in speed occurs, using an ad-hoc colormap changing from green (slow) to red (fast). Following the procedure described in [11], the trajectory is analyzed to look for possible temporal breakpoints, defined as sudden stops and re-starts. Such discontinuities happen when the position of the object of interest at the beginning and end of a defined time interval $\left[t_{k}, t_{k+1}\right]$ is within a predefined range $\Delta$. Mathematically, and according to [11], this implies:

$$
P_{k+1}^{i}=P_{k}^{i} \pm \Delta \quad \text { where } \quad P_{k}^{i}=\left(x_{k}^{i}, z_{k}^{i}, t_{k}\right)
$$

A temporal breakpoint is inserted when, within a second, the position of the wheelchair varies less than $30 \mathrm{~cm}$. In [11], also spatial breakpoints are extracted to describe the trajectory, defined as sudden variations of the motion direction. This means computing the angle between two segments of 


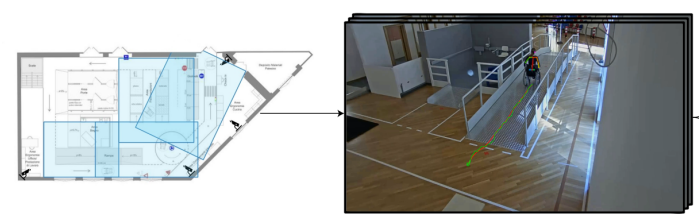

Configuration Synchronous recording
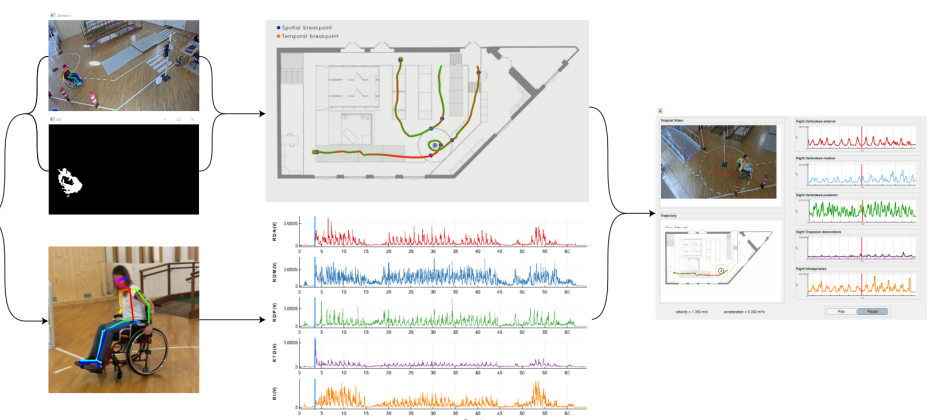

Joint trajectory and EMG analysis

Fusion

Figure 3: Pipeline of the proposed method: sensor positioning, camera calibration, video recording, real-time trajectory analysis, posture and arm fatigue analysis

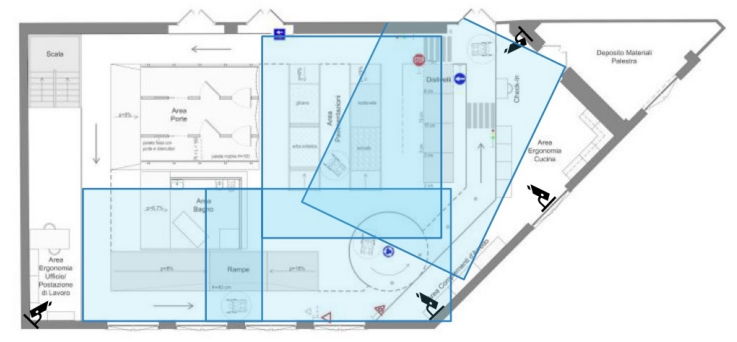

Figure 4: Layout of the AUSILIA gym. The environment is currently covered by four IP cameras, necessary to monitor the wheelchair path. The approximate coverage of the cameras is highlighted in light blue.

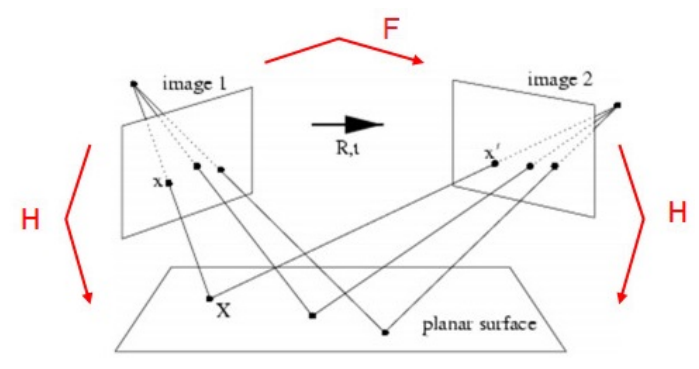

Figure 5: Computation of the fundamental and homography matrices

the trajectory in order to search for sharp direction variations:

$$
\begin{gathered}
\beta_{k}=\left|\frac{m_{k-1}-m_{k}}{1-m_{k-1} m_{k}}\right|>\beta_{t h} \\
\text { where }\left\{\begin{array}{l}
r_{k-1}(x)=m_{k-1} x+q_{k-1} \\
r_{k}(x)=m_{k} x+q_{k}
\end{array}\right.
\end{gathered}
$$

In our case, we use a temporal reference of two seconds

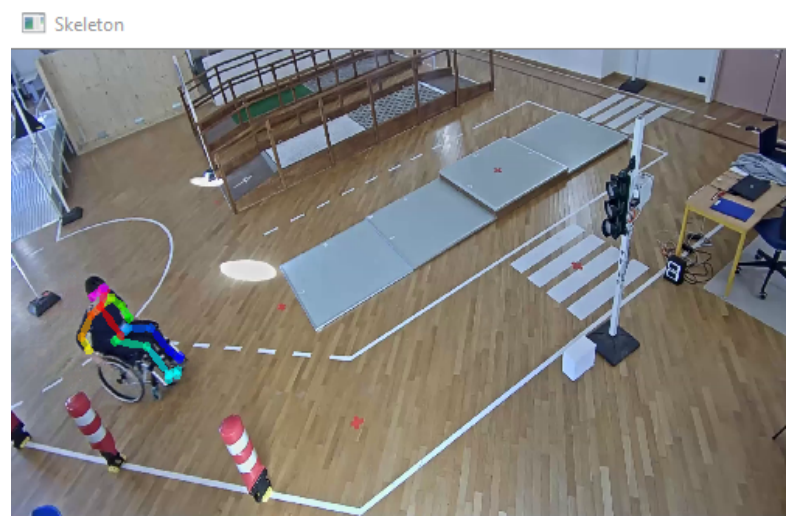

(a) Extraction of the skeleton through OpenPose [4]

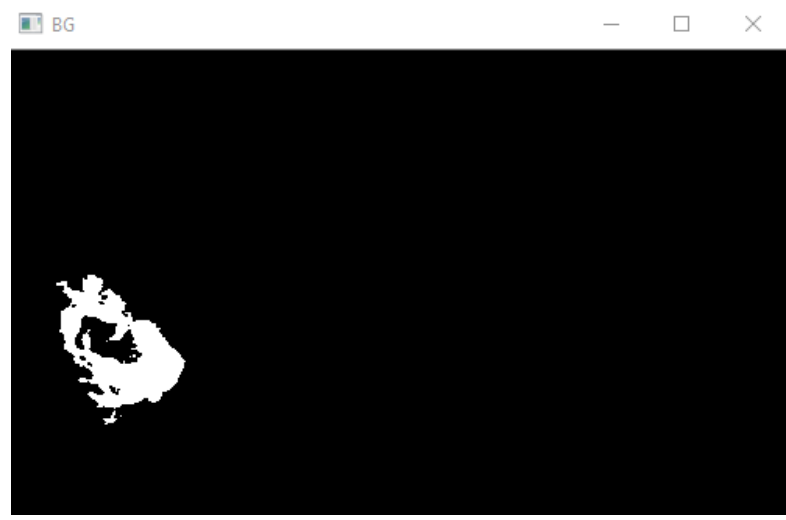

(b) Motion detection via background subtraction

Figure 6: Example of skeleton extraction and motion detection through background subtraction.

in order to calculate the angle of rotation performed by the users at a precise instant. 


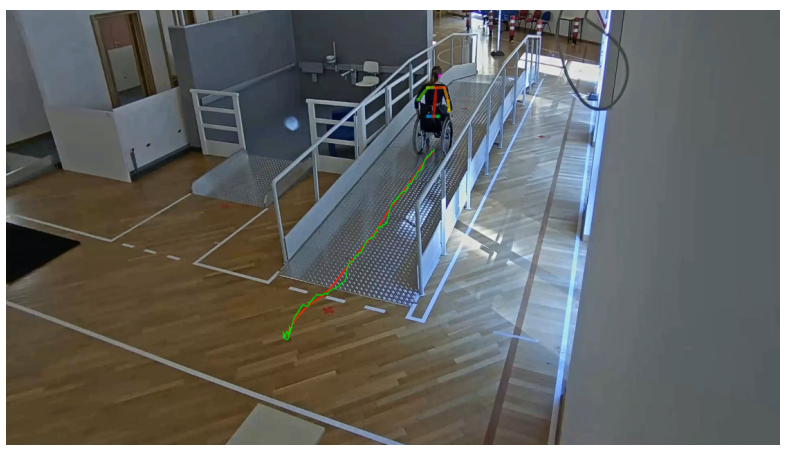

(a) Smoothed trajectory in the real world

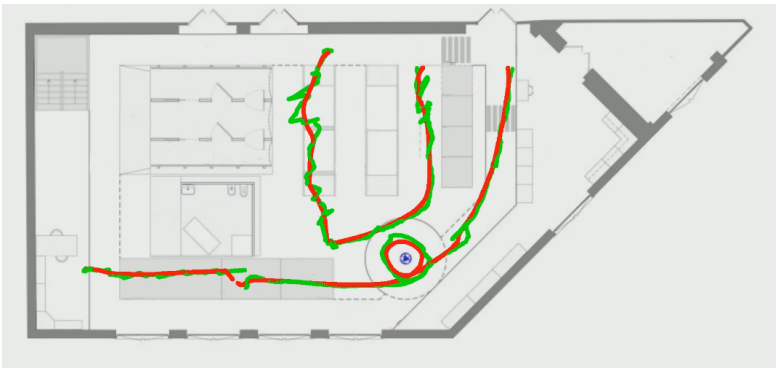

(b) Projected smoothed trajectory

Figure 7: Trajectory filtering: the green line represents the output of the Kalman filter, while the red line is the result of the moving average filter.

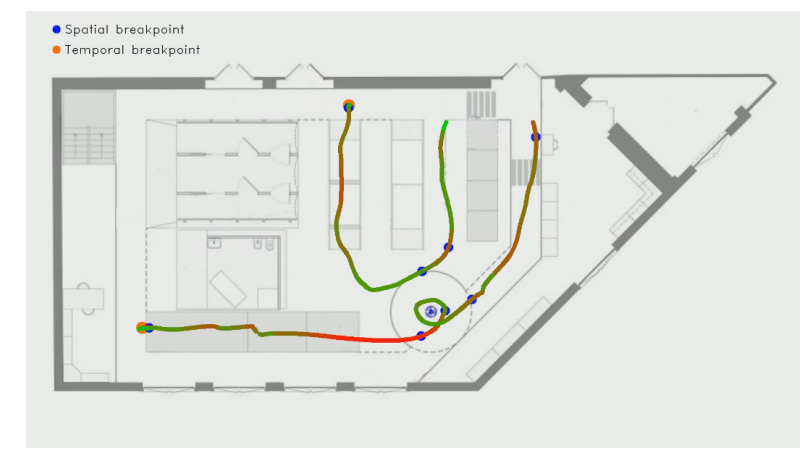

Figure 8: Trajectory performed by a test user projected on the gym map showing: speed changes, spatial breakpoints and temporal breakpoints.

\subsection{Shoulder forces and posture analysis}

In addition to the extraction of the trajectory of the wheelchair, our aim is to also study the posture and the arm movement while pushing the wheelchair. In particular, the shoulder movement is analyzed in detail as the prolonged use of wheelchairs with manual self-propulsion causes shoulder disorders in most users. As a consequence, the pushing force distribution is analyzed to understand if there are discontinuities that, in the long period, can be a potential source of pain. To achieve this second objective, several tools have been used: five EMG sensors to detect the muscular activity of the shoulder, the angle of the elbow extracted from the skeleton, and an electrogoniometer, mostly used as a ground truth to measure the reliability of the OpenPose joint extraction. Each user is asked to test three different types of wheelchairs (Fig. 11). The EMG and the electrogoniometer are used to directly analyze the user's movement. The motivation for using three different wheelchairs, instead, is necessary to evaluate the performances while changing on the one hand the weight of the wheelchair itself, and on the other hand the responsiveness of the wheelchair when a force is applied to the wheels. This second difference mostly depends on the different inclination of the seat and, consequently, the different posture of the user. For this reason, in addition to the analysis of the shoulder, the objective is also to understand if such different postures can help in preventing shoulder pain.

The most significant data for the study of user movement, and in particular the shoulder movement, is obtained from the EMG sensors. A common framework to analyze these data is MOKKA 2 which is is provided by the opensource Biomechanical ToolKit (BTK) ${ }^{3}$

The EMG sensors measure the muscle activity, including both voluntary and involuntary movements performed by muscle fibers.

The amplitude of the signal is analyzed in the time domain, as it reflects the force used by the muscle, i.e. its muscle activation. In the frequency domain, it is possible to extract values related to the fatigue.

In our implementation the EMG signals have been analyzed and processed in the time domain through the following steps (Fig. 9): data extraction, filtering, rectification and RMS (Root Mean Square) envelope.

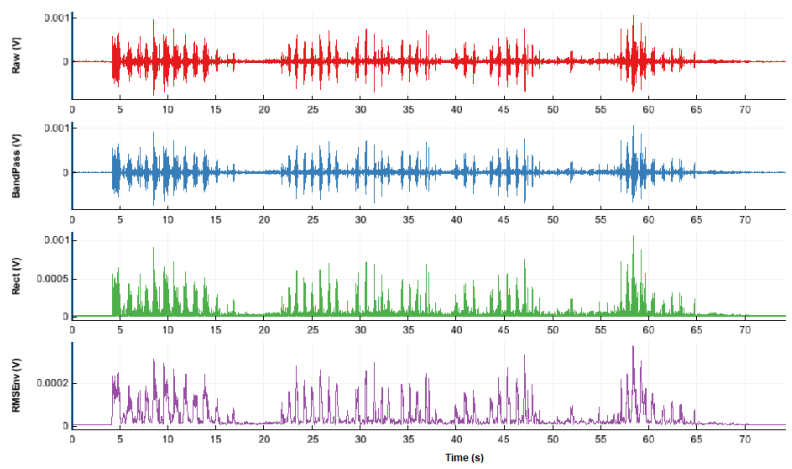

Figure 9: EMG signal processing: raw signal (red), bandpass filtered signal (blue), rectified signal (green) and the RMS envelope (violet). The signal amplitude is reported in Volt.

\footnotetext{
2 http://biomechanical-toolkit.github.io/mokka/

${ }^{3}$ https://code.google.com/archive/p/b-tk/
} 
Since the signal analyzed in the time domain is a representation of the muscular force, that is the muscular activation, it is possible to define the mean muscle activation by computing the mean of the signal. This average value is extracted for every signal and for every trial using the different wheelchair. Consequently, by calculating the ratio between the average values of each signal passing from one wheelchair to another, it is possible to understand if and how the muscular activation changes if the seat is changed.

Together with the EMG information, the angle of opening and closing of the elbow is also extracted, to have an additional data on the control on the movement of the arm. However, the electrogoniometer is rather invasive and we preferred using the joints of the skeleton, providing a weak replacement for the electrogoniometer data, for a more comfortable experience for the user, who will not be required to wear invasive sensors during the experiments. From the skeleton extracted at each frame, the positions $(x, y)$ of three joints are extracted: the shoulder, the elbow and the wrist. These positions are stored so that they can be used and processed later to evaluate the variations of the angle at the elbow level. The procedure used for the calculation of the opening and closing of the elbow, relies on the same strategy adopted for the identification of spatial and temporal breakpoints in the trajectory analysis.

\subsection{Results correlation and displaying}

At this point of the study, the data is processed, and can be viewed by the medical personnel in order to correlate the information coming from the different information sources, bearing in mind that each source has different temporal resolutions requiring an additional effort in synchronization. An overview of the final layout of the interface is shown in Fig. $10 \mathrm{~b}$

\section{Results}

The main objective of this study is to find a correlation between the development of shoulder pain in users and the prolonged or incorrect usage of the wheelchair. Considered that the study presented is pioneering in its nature and not a proper clinical study, we involved six healthy users of different age, musculature and gender, as shown in Table 1 . In the considered environment, users are subject to clinical trials and are constantly monitored by therapists and nursing staff on a daily basis, in order to optimize the choice of the most suitable wheelchair.

In the tests, users were asked to perform an established route three times, each time using a different wheelchair model. At the end of each trial, users were required to fill in a questionnaire to collect their direct feedback about the level of fatigue.

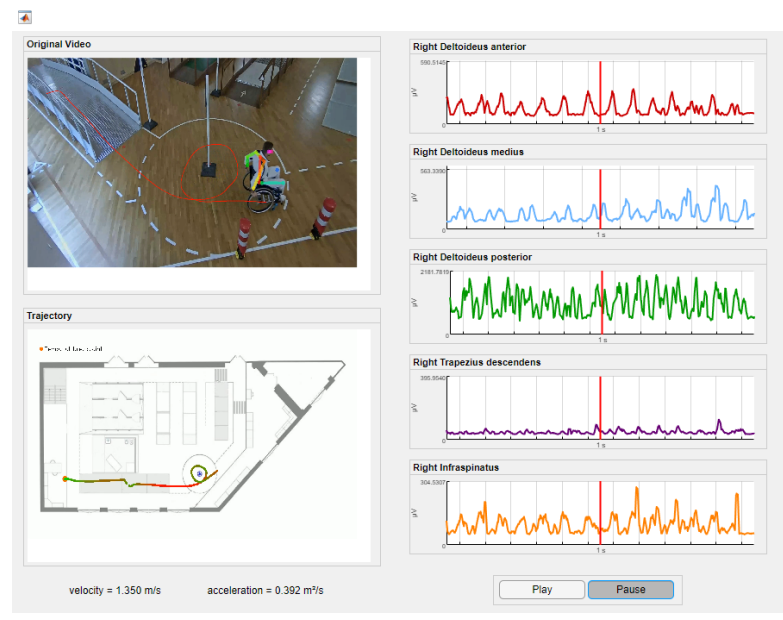

(a) Graphical interface

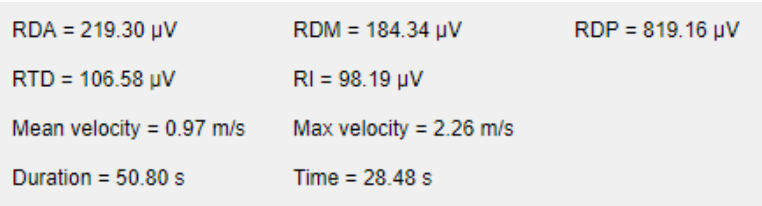

(b) An example of extracted data

Figure 10: Graphical interface developed to display all data at the same time. A play / pause button is present to be able to observe in detail all the signals at a precise moment. The displayed data includes the mean value of muscular activation for every EMG sensor, the mean and maximum velocity of the user as well as the entire duration of the trial.

\begin{tabular}{l|c|c|c}
\hline \hline & Gender & Musculature & $\begin{array}{c}\text { Experience } \\
\text { with wheelchairs }\end{array}$ \\
\hline \hline User 1 & male & well-developed & 3 \\
\hline User 2 & female & normal & 2 \\
\hline User 3 & female & normal & 1 \\
\hline User 4 & female & normal & 4 \\
\hline User 5 & male & normal & 2 \\
\hline User 6 & female & normal & 1
\end{tabular}

Table 1: Users involved in the experiments, with experience level between 1 (beginner) and 5 (expert).

\subsection{Trajectory analysis}

For what concerns the trajectory analysis, the obtained results confirm our initial hypotheses, showing how the speed and the motion properties of users change in presence of obstacles and turns, while maximum speed is reached in presence of straight paths and descents. An example of the salient interest points, represented through the temporal and spatial breakpoints, can be seen in Fig. 12. It is shown how User 6, with a Level 1 (beginner) wheelchair 


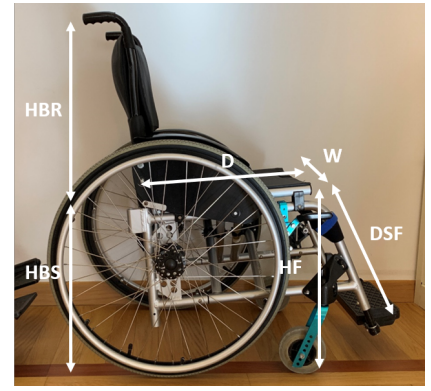

(a) "Vega": super light, responsive.

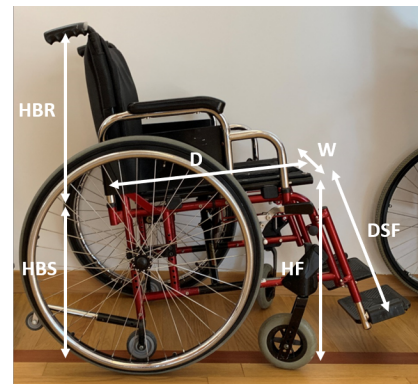

(b) "Ministar": light, responsive.

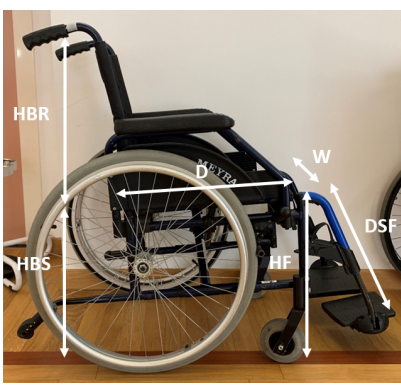

(c) "Meyra": light.

Figure 11: Characteristics of the three types of wheelchair, which vary in terms of weight and responsiveness.

usage experience, encountered severe difficulties in facing obstacles in the pre-determined path, as also highlighted by the breakpoints plot in Fig. $12 \mathrm{~b}$. The user also encounters difficulties while getting on the ramp in Fig. 12a. To continue along the path, the user suddenly changes the motion direction to reach for the handrail, which was not in range along the path. We can notice that a set of useful information can be inferred from the trajectory plot in $12 \mathrm{~b}$, in particular: (i) the speed has decreased significantly (light green) during the ascent, as shown by the presence of temporal breakpoints; (ii) when the user continues the test with the help of the handrail, a spatial interruption is drawn on the map due to an abrupt change in direction. Such breakpoints are successfully detected by our trajectory analysis and they also match the answers that users gave in the questionnaire. Moreover, users identified the climbs, descents and also the different terrain types as the most difficult parts along the path, which is in line with the data observed in our arm movement and posture analysis.

From the analysis it turned out that the results of the trajectory analysis in relation with the different wheelchair models were less relevant than expected. By changing the wheelchair model from the most responsive to the less responsive one (Fig. 11, a progressive increase in the employed force was expected, associated to a decrease in speed. However, this trend was not evident in every test, as can be seen in Fig. 13. This might be due to the fact that users have not been pushed to maximum strain, and only a limited number of trials has been performed. However, despite the small number of samples used to perform the analysis, both the quantitative results and the user questionnaires show how the wheelchair weight is not the main factor linked to speed variations along the path. In fact, from such a preliminary analysis, it appears from the trajectory data, that the inclination of the wheelchair along the path has a far more relevant influence than the weight variation of the wheelchair itself.

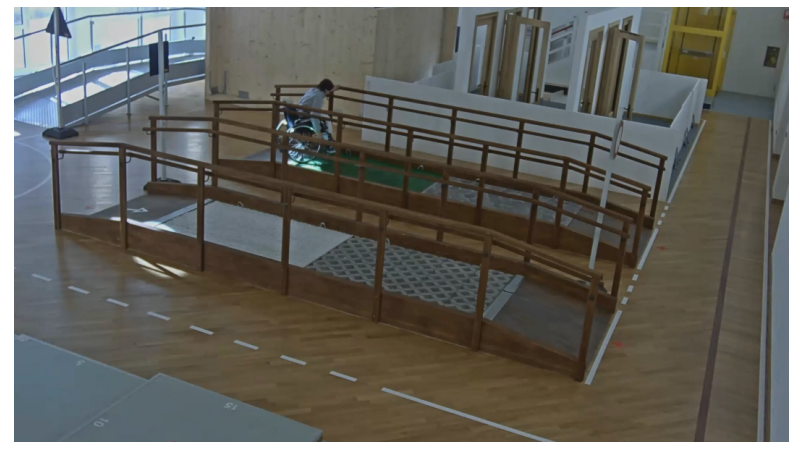

(a) User 6 that stops on the ascent and continue the path clings to the parapet

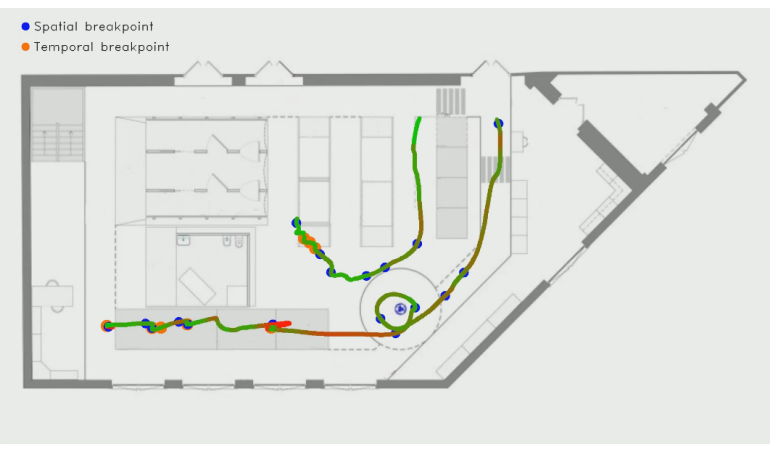

(b) Temporal and spatial breakpoints on the map caused by the difficulties encountered

Figure 12: Example of difficulty caused by obstacles (user $6)$.

\subsection{Arm movement and posture analysis}

We conducted a similar analysis also for the shoulder movement and user posture, relating them with the presence of obstacles along the path and the wheelchair type. As regards the comparison with the different types of wheelchair, we calculate the mean value $\mu_{p}: p \in P$ of each users muscular activity, as extracted by the EMG sensor, and compare 


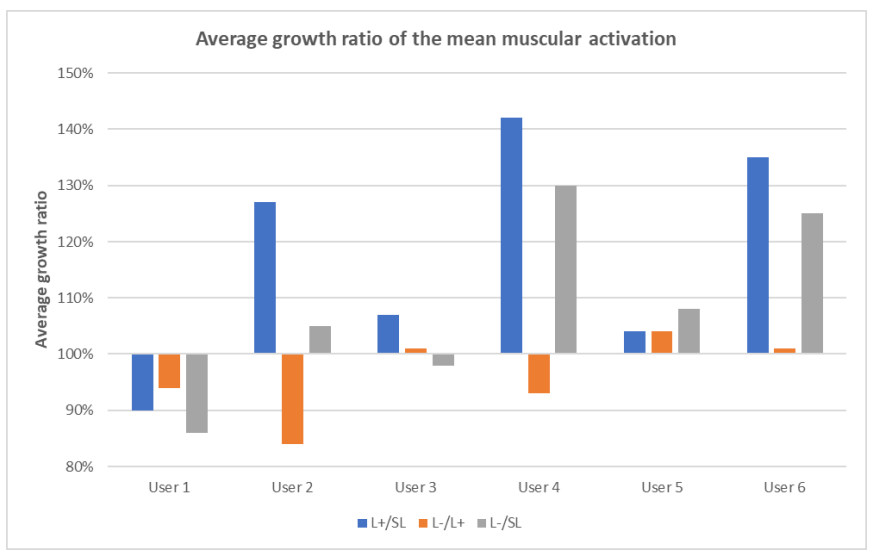

Figure 13: Average growth ratio of the mean muscular activation for the 6 users on the three wheelchair models. Notice how the muscular fatigue changes based on user experience and how in our tests it is not strictly linked just to the wheelchair weight.

with the rest of the user population $P$. Then, by calculating the ratio of the mean value in passing from one wheelchair model to the other, it was possible to quantify the change in the force applied $\Delta_{f}$, as performed by the user. The data obtained from this analysis are shown in Table 2. Also in this case the idea is that when the wheelchair changes, the users force increases proportionally to the difficulty of the path. During the evaluation of these parameters, while speed seems to be more related to the wheelchair inclination, the required force is more related to weight. In fact, an increase in muscle activity occurred for five out of six users, when switching from a super-light to a light wheelchair, but both tilted. Instead, only half of the users in the panel has increased the muscular force by varying the inclination of the seat.

\subsection{Results correlation}

By visualizing and integrating all the data together through the developed graphical interface (Fig. 10a), it is possible to have a complete and detailed view of the entire trial. Through the video and the projection of the trajectory on the gym map, it is possible to observe when and where the user's difficulties occur, and understand how specific obstacles in the path can lead to an increasing fatigue. At the same time, in addition to visualizing spatial and temporal breakpoints in the path thanks to the trajectory analysis, it is possible to also visually correlate the EMG signals of each muscle at every precise moment. Observing the signal during the various phases of the route, it is possible to notice how, in presence of situations considered critical by the users (ascent, as well as different surfaces like grass and stone), muscle activity increases with numerous

\begin{tabular}{c|c|c|c}
\hline \hline & L+/SL & L-/L+ & L-/SL \\
\hline \hline User 1 & $90 \%$ & $94 \%$ & $86 \%$ \\
\hline User 2 & $127 \%$ & $84 \%$ & $105 \%$ \\
\hline User 3 & $107 \%$ & $101 \%$ & $98 \%$ \\
\hline User 4 & $142 \%$ & $93 \%$ & $130 \%$ \\
\hline User 5 & $104 \%$ & $104 \%$ & $108 \%$ \\
\hline User 6 & $135 \%$ & $101 \%$ & $125 \%$
\end{tabular}

Table 2: Average growth ratio of the mean muscular activation between pairs of wheelchairs: super light, responsive $(S L)$, light, responsive $(L+)$, light $(L-)$. A $100 \%$ ratio indicates that with the second wheelchair the user employed $100 \%$ of the average force used with the previous wheelchair, therefore a constant muscle activity (ratio equal to 1 ). While a $90 \%$ ratio indicates that with the second wheelchair only $90 \%$ of the previous force was used, i.e. there was a $10 \%$ decrease. A value greater than $100 \%$ therefore indicates an increase in muscle activity.

peaks. This allows the medical personnel to improve their knowledge about the user's fatigue, which would be otherwise difficult to gather, clearly showing the benefits of having a distributed sensing framework like the one available in AUSILIA.

\section{Conclusions}

In this paper, we propose an approach for joint trajectory and fatigue analysis in wheelchair users. We combine the state-of-the-art skeleton-based pose estimation techniques, classic computer vision algorithms and data from wearable sensors to provide the medical personnel with a complete solution that enables them to evaluate an ensemble of parameters jointly, while performing a pre-defined task. Our study shows that a relationship exists between the trajectory data and the EMG signals, both in terms of velocity, and temporal and spatial breakpoints. Furthermore, our method takes into account different wheelchairs, showing how many different factors, including the user experience and the terrain slope and type of surface, all contribute to the user's fatigue at different levels.

This confirms that the setup we have envisaged and the multi-sensory environment we have defined can indeed be of help in correlating the muscular activity to the trajectory. We believe that the main limitation we have been facing so far is mostly due to the small number of users involved and trials performed. This issue is currently being addressed via the definition of an ad-hoc clinical study.

\section{References}

[1] M. Alm, H. Saraste, and C. Norrbrink. Shoulder pain in persons with thoracic spinal cord injury: prevalence and charac- 
teristics. Journal of rehabilitation medicine, 40(4):277-283, 2008.

[2] L. R. Altimari, J. L. Dantas, M. Bigliassi, T. F. D. Kanthack, A. C. de Moraes, and T. Abrão. Influence of different strategies of treatment muscle contraction and relaxation phases on emg signal processing and analysis during cyclic exercise. In Computational Intelligence in Electromyography Analysis-A Perspective on Current Applications and Future Challenges. IntechOpen, 2012.

[3] A. Bewley, Z. Ge, L. Ott, F. Ramos, and B. Upcroft. Simple online and realtime tracking. In 2016 IEEE International Conference on Image Processing (ICIP), pages 3464-3468, 2016.

[4] Z. Cao, G. Hidalgo, T. Simon, S.-E. Wei, and Y. Sheikh. Openpose: realtime multi-person $2 \mathrm{~d}$ pose estimation using part affinity fields. arXiv preprint arXiv:1812.08008, 2018.

[5] Z. Cao, T. Simon, S.-E. Wei, and Y. Sheikh. Realtime multiperson $2 \mathrm{~d}$ pose estimation using part affinity fields. In CVPR, 2017.

[6] K. Curtis, K. Roach, E. B. Applegate, T. Amar, C. Benbow, T. Genecco, and J. Gualano. Development of the wheelchair user's shoulder pain index (wuspi). Spinal Cord, 33(5):290, 1995.

[7] K. A. Curtis, G. A. Drysdale, R. D. Lanza, M. Kolber, R. S. Vitolo, and R. West. Shoulder pain in wheelchair users with tetraplegia and paraplegia. Archives of physical medicine and rehabilitation, 80(4):453-457, 1999.

[8] C. J. De Luca, L. D. Gilmore, M. Kuznetsov, and S. H. Roy. Filtering the surface emg signal: Movement artifact and baseline noise contamination. Journal of biomechanics, 43(8): 1573-1579, 2010.

[9] M. A. Finley and M. M. Rodgers. Prevalence and identification of shoulder pathology in athletic and nonathletic wheelchair users with shoulder pain: A pilot study. Journal of Rehabilitation Research \& Development, 41, 2004.

[10] J. L. Mercer, M. Boninger, A. Koontz, D. Ren, T. DysonHudson, and R. Cooper. Shoulder joint kinetics and pathology in manual wheelchair users. Clinical Biomechanics, 21(8):781-789, 2006.

[11] N. Piotto, N. Conci, and F. G. De Natale. Syntactic matching of trajectories for ambient intelligence applications. IEEE Transactions on Multimedia, 11(7):1266-1275, 2009.

[12] T. Pisoni, N. Conci, F. G. De Natale, M. De Cecco, G. Nollo, A. Frattari, and G. M. Guandalini. Ausilia: assisted unit for simulating independent living activities. In Smart Cities Conference (ISC2), 2016 IEEE International, pages 1-4. IEEE, 2016.

[13] A. W. Smeulders, D. M. Chu, R. Cucchiara, S. Calderara, A. Dehghan, and M. Shah. Visual tracking: An experimental survey. IEEE transactions on pattern analysis and machine intelligence, 36(7):1442-1468, 2013.

[14] M. L. Systems. Motion lab systems- a software user guide for emg graphing and emg analysis. In 2018 IEEE International Conference on Robotics and Automation (ICRA), pages 1145. Motion Lab Systems, 2009.

[15] A. Yilmaz, O. Javed, and M. Shah. Object tracking: A survey. Acm computing surveys (CSUR), 38(4):13, 2006.
[16] T. N. S. T. Zawawi, A. R. Abdullah, M. H. Jopri, T. Sutikno, N. M. Saad, and R. Sudirman. A review of electromyography signal analysis techniques for musculoskeletal disorders. Indonesian Journal of Electrical Engineering and Computer Science, 11(3):1136-1146, 2018.

[17] C. Zimmermann, T. Welschehold, C. Dornhege, W. Burgard, and T. Brox. 3d human pose estimation in rgbd images for robotic task learning. In 2018 IEEE International Conference on Robotics and Automation (ICRA), pages 1986-1992. IEEE, 2018. 